\title{
Xanthine Oxidoreductase Activity Is Correlated With Hepatic Steatosis
}

\section{Chisako Yagi}

Hyogo College of Medicine

Yoshiki Kusunoki ( $\nabla$ ykusu@hyo-med.ac.jp )

Hyogo College of Medicine

\section{Taku Tsunoda}

Hyogo College of Medicine

\section{Takayo Murase}

Sanwa Kagaku Kenkyusho

\section{Takashi Nakamura}

Sanwa Kagaku Kenkyusho

\section{Keiko Osugi}

Hyogo College of Medicine

\section{Mana Ohigashi}

Hyogo College of Medicine

\section{Akiko Morimoto}

Hyogo College of Medicine Akio Miyoshi

Hyogo College of Medicine

Miki Kakutani-Hatayama

Hyogo College of Medicine

\section{Kae Kosaka-Hamamoto}

Hyogo College of Medicine

Manabu Kadoya

Hyogo College of Medicine

\section{Kosuke Konishi}

Hyogo College of Medicine

\section{Takuhito Shoji}

Hyogo College of Medicine Hidenori Koyama

Hyogo College of Medicine

\section{Research Article}


Keywords: XOR, UA, quadrupole mass spectrometry, hyperuricemia, liver-to-spleen

Posted Date: September 17th, 2021

DOI: https://doi.org/10.21203/rs.3.rs-882776/v1

License: (c) (1) This work is licensed under a Creative Commons Attribution 4.0 International License. Read Full License 


\section{Abstract}

The enzyme xanthine oxidoreductase (XOR) catalyzes the formation of uric acid (UA) from hypoxanthine and xanthine, which in turn are products of purine metabolism starting from ribose-5-phosphate. Several studies suggested a relationship between hyperuricemia and hepatic steatosis; however, few previous studies have directly examined the relationship between XOR and hepatic steatosis. A total of 223 subjects with one or more cardiovascular risk factors were enrolled. Hepatic steatosis was calculated according to the liver-to-spleen (L/S) ratio on computed tomography and the hepatic steatosis index (HSI). We measured a plasma XOR activity assay using a newly established highly sensitive assay based on $\left[{ }^{13} \mathrm{C}_{2},{ }^{15} \mathrm{~N}_{2}\right]$ xanthine and liquid chromatography/triple quadrupole mass spectrometry. XOR activity and the UA level were increased in subjects with $\mathrm{L} / \mathrm{S}$ ratio $<1.1$ and $\mathrm{HSI}<36$. Multivariate logistic regression analysis indicated that plasma XOR activity was associated with the risk of hepatic steatosis as assessed by the L/S ratio and HSI independently of insulin resistance, whereas UA levels were not associated with risk of hepatic steatosis. The results of this study indicated that plasma XOR activity is associated with hepatic steatosis independently of insulin resistance and serum UA levels.

\section{Background}

Nonalcoholic fatty liver disease (NAFLD) is defined as the presence of fatty accumulation in the liver on imaging or histology, excluding fatty liver secondary to alcohol, drugs, or genetic disorders [1, 2]. NAFLD is a major cause of liver disease, and its prevalence is reported to be increasing [1-3]. NAFLD has been reported to be a risk factor for not only hepatic diseases-related mortality but also cardiovascular disease [4-7].

Imaging techniques such as abdominal ultrasonography, computed tomography (CT), and magnetic resonance imaging are useful in the evaluation of hepatic steatosis. Abdominal ultrasound is the recommended screening test for NAFLD, but CT has also been used to evaluate hepatic steatosis. The CT value of the liver decreases with the degree of fat deposition, and by measuring the ratio of the CT values in the liver and to those in the spleen [the liver-to-spleen (L/S) ratio], it is possible to calculate the fat content of the liver [8-12]. In addition to imaging tests, the hepatic steatosis index (HSI) has been reported to be useful as screening indices for hepatic steatosis [13].

Metabolic syndrome and type 2 diabetes mellitus, which are associated with insulin resistance, are known to be risk factors for the development of NAFLD [14-16]. In addition, hyperuricemia has been reported to be a risk factor for metabolic syndrome and NAFLD $[17,18]$. Xanthine oxidoreductase (XOR) is an enzyme regulating synthesis of uric acid (UA) and generation of reactive oxygen species $[19,20]$. XOR activity is associated with insulin resistance [21], and it has been reported that XOR activity is elevated in metabolic syndrome and type 2 diabetes $[22,23]$. In addition, previous studies demonstrated that XOR activity was significantly increased in a mouse model of NAFLD and that fatty liver induced by a high-fat diet was suppressed by administration of XOR inhibitors [24, 25]. 
It is assumed that XOR activity is associated with insulin resistance and hepatic steatosis in humans. However, in humans, XOR activity is extremely low compared with that in rodents; this makes accurate measurement difficult [26-28]. A novel human plasma XOR activity assay has been established using a combination of liquid chromatography (LC) and triple quadrupole mass spectrometry (TQMS) to detect $\left[{ }^{13} \mathrm{C}_{2},{ }^{15} \mathrm{~N}_{2}\right]$ UA using $\left[{ }^{13} \mathrm{C}_{2},{ }^{15} \mathrm{~N}_{2}\right]$ xanthine as a substrate [26-28].

The aim of this study was to clarify the relationship between XOR activity accurately evaluated by this novel method and hepatic steatosis assessed by the L/S ratio and HSI in humans.

\section{Results}

\section{Characteristics of the study participants}

Results are given as median (interquartile range), unless otherwise stated. The characteristics of the participants are presented in Table 1. There were 223 subjects, consisting of 142 females and 81 males. The age was 66 (53-73) years; the body mass index (BMI) was $23.1(21.2-26.4) \mathrm{kg} / \mathrm{m}^{2}$, the abdominal circumference (AC) assessed using CT was 83.9 (77.0-91.8) cm, subcutaneous fat area (SFA) was 162.7 (108.1-221.8) $\mathrm{cm}^{2}$, and visceral fat area (VFA) was $86.1(53.1-119.0) \mathrm{cm}^{2}$. XOR activity was $42.7(25.3-$ 78.6) $\mathrm{pmol} / \mathrm{h} / \mathrm{mL}$; serum UA was $5.3(4.5-6.2) \mathrm{mg} / \mathrm{dL}$; the L/S ratio was 1.3 (1.2-1.5); and HSI was 32.6 (29.3-37.9). 
Table 1

Participant characteristics

\begin{tabular}{|ll|}
\hline N (Female : Male) & $223(142: 81)$ \\
\hline Age (years) & $66(53-73)$ \\
\hline BMI $\left(\mathrm{kg} / \mathrm{m}^{2}\right)$ & $23.1(21.2-26.4)$ \\
\hline Abdominal circumference $(\mathrm{cm})$ & $83.9(77.0-90.8)$ \\
\hline Subcutaneous fat area $\left(\mathrm{cm}^{2}\right)$ & $162.7(108.1-221.8)$ \\
\hline Visceral fat area $\left(\mathrm{cm}^{2}\right)$ & $86.1(53.1-119.0)$ \\
\hline HbA1c $(\%)$ & $5.8(5.6-6.2)$ \\
\hline HOMA-R & $1.4(0.9-2.2)$ \\
\hline T-Chol (mg/dL) & $194.0(174.0-215.0)$ \\
\hline TG (mg/dL) & $108.0(77.0-154.5)$ \\
\hline HDL-Chol (mg/dL) & $57.0(48.5-70.0)$ \\
\hline XOR activity (pmol/h/mL) & $42.7(25.3-78.6)$ \\
\hline UA (mg/dL) & $5.3(4.5-6.2)$ \\
\hline L/S ratio & $1.3(1.2-1.5)$ \\
\hline HSI & $32.6(29.3-37.9)$ \\
\hline AST (U/L) & $20.0(16.0-25.0)$ \\
\hline ALT (U/L) & $18.0(13.0-26.0)$ \\
\hline Y-GTP & $21.0(15.8-32.0)$ \\
\hline Hypertension & $171(76.7 \%)$ \\
\hline Diabetes & $51(22.9 \%)$ \\
\hline Dyslipidemia & $145(65.0 \%)$ \\
\hline
\end{tabular}

The results are presented as median (interquartile range). BMI, body mass index; $\mathrm{HbA1c}$, hemoglobin A1c; HOMA-R, homeostasis model assessment ratio; T-Chol, total-cholesterol; TG, triglycerides; HDL-Chol, highdensity lipoprotein-cholesterol; XOR, xanthine oxidoreductase; UA, uric acid; L/S, liver-to-spleen; HSI, hepatic steatosis index; AST, aspartate transaminase; ALT, alanine transaminase: $\gamma$-GTP, $\gamma$-glutamyl transpeptidas.

Differences in patient background categorized by XOR activity and UA levels 
Participants were divided into quartiles based on XOR activity (Table 2). BMI, AC, SFA, and VFA tended to increase with increasing XOR activity: quartile 1 with the lowest and quartile 4 with the highest XOR activity. HbA1c, homeostasis model assessment ratio (HOMA-R), aspartate transaminase (AST), alanine aminotransferase (ALT), and $\gamma$-glutamyl transpeptidase ( $\gamma$-GTP) also tended to increase with increasing XOR activity. The proportion of the $\mathrm{L} / \mathrm{S}$ ratio $<1.1$ and $\mathrm{HSI}>36.0$ for each XOR activity is illustrated in Fig.

1. The results indicated that the proportion of patients with $\mathrm{L} / \mathrm{S}$ ratio $<1.1$ and $\mathrm{HSI}>36.0$ tended to increase with increasing XOR activity. 
Table 2

Differences in clinical parameters among quadrants of xanthine oxidoreductase (XOR) activities

\begin{tabular}{|c|c|c|c|c|c|}
\hline $\begin{array}{l}\text { XOR } \\
\text { Variables }\end{array}$ & $\begin{array}{l}\text { Quartile } 1 \\
(N=56)\end{array}$ & $\begin{array}{l}\text { Quartile } 2 \\
(N=55)\end{array}$ & $\begin{array}{l}\text { Quartile } 3 \\
(N=56)\end{array}$ & $\begin{array}{l}\text { Quartile } 4 \\
(N=56)\end{array}$ & $\begin{array}{l}P \text { for } \\
\text { trend }\end{array}$ \\
\hline $\begin{array}{l}\text { Female : } \\
\text { Male }\end{array}$ & $44: 12$ & $42: 13$ & $26: 30$ & $30: 26$ & $<0.001$ \\
\hline Age (years) & $65(52-75)$ & $69(65-74)$ & $62(50-71)$ & $62(53-71)$ & 0.080 \\
\hline $\mathrm{BMI}\left(\mathrm{kg} / \mathrm{m}^{2}\right)$ & $\begin{array}{l}21.5(20.3- \\
23.3)\end{array}$ & $\begin{array}{l}23.0(21.5- \\
24.9)\end{array}$ & $\begin{array}{l}24.0(21.8- \\
27.1)\end{array}$ & $\begin{array}{l}26.0(22.9- \\
29.4)\end{array}$ & $<0.001$ \\
\hline $\mathrm{AC}(\mathrm{cm})$ & $\begin{array}{l}77.4(71.9- \\
85.3)\end{array}$ & $\begin{array}{l}83.1(78.5- \\
87.5)\end{array}$ & $\begin{array}{l}84.1(76.9- \\
92.4)\end{array}$ & $\begin{array}{l}88.9(84.2- \\
99.2)\end{array}$ & $<0.001$ \\
\hline $\mathrm{SFA}\left(\mathrm{cm}^{2}\right)$ & $\begin{array}{l}122.2(82.8- \\
174.3)\end{array}$ & $\begin{array}{l}165.6(126.8- \\
203.9)\end{array}$ & $\begin{array}{l}160.2(105.1- \\
232.1)\end{array}$ & $\begin{array}{l}193.7(138.6- \\
254.7)\end{array}$ & $<0.001$ \\
\hline VFA $\left(\mathrm{cm}^{2}\right)$ & $\begin{array}{l}54.4(27.6- \\
87.3)\end{array}$ & $\begin{array}{l}81.1(53.1- \\
108.4)\end{array}$ & $\begin{array}{l}82.1(58.3- \\
113.3)\end{array}$ & $\begin{array}{l}119.8(90.8- \\
156.0)\end{array}$ & $<0.001$ \\
\hline HbA1c (\%) & $5.6(5.4-5.9)$ & $5.8(5.5-6.2)$ & $5.7(5.6-6.2)$ & $6.1(5.8-6.9)$ & $<0.001$ \\
\hline HOMA-R & $1.1(0.7-1.6)$ & $1.2(0.9-1.7)$ & $1.7(1.1-2.1)$ & $2.1(1.4-2.8)$ & $<0.001$ \\
\hline $\begin{array}{l}\text { T-Chol } \\
\text { (mg/dL) }\end{array}$ & $\begin{array}{l}190.0(174.8- \\
209.0)\end{array}$ & $\begin{array}{l}191.0(177.0- \\
214.0)\end{array}$ & $\begin{array}{l}198.5(174.8- \\
215.0)\end{array}$ & $\begin{array}{l}\text { 194.5 (169.5- } \\
217.0)\end{array}$ & 0.383 \\
\hline TG (mg/dL) & $\begin{array}{l}81.5(63.8- \\
126.3)\end{array}$ & $\begin{array}{l}108.0(75.0- \\
130.0)\end{array}$ & $\begin{array}{l}109.0(85.0- \\
159.5)\end{array}$ & $\begin{array}{l}130.5(98.5- \\
199.3)\end{array}$ & $<0.001$ \\
\hline $\begin{array}{l}\text { HDL-Chol } \\
\text { (mg/dL) }\end{array}$ & $\begin{array}{l}66.0(55.0- \\
76.5)\end{array}$ & $\begin{array}{l}63.0(52.5- \\
75.0)\end{array}$ & $\begin{array}{l}53.5(48.0- \\
64.5)\end{array}$ & $\begin{array}{l}50.0(43.8- \\
58.0)\end{array}$ & $<0.001$ \\
\hline $\begin{array}{l}\text { XOR activity } \\
\text { (pmol/h/mL) }\end{array}$ & $\begin{array}{l}18.0(14.4- \\
21.0)\end{array}$ & $\begin{array}{l}31.4(27.5- \\
34.6)\end{array}$ & $\begin{array}{l}59.1(49.3- \\
70.0)\end{array}$ & $\begin{array}{l}114.5(90.5- \\
179.0)\end{array}$ & $<0.001$ \\
\hline $\mathrm{UA}(\mathrm{mg} / \mathrm{dL})$ & $4.8(4.0-5.8)$ & $5.0(4.4-5.6)$ & $5.4(4.7-6.1)$ & $5.8(5.0-6.5)$ & $<0.001$ \\
\hline L/S ratio & $1.4(1.3-1.5)$ & $1.4(1.3-1.5)$ & $1.3(1.2-1.5)$ & $1.2(1.0-1.3)$ & $<0.001$ \\
\hline $\mathrm{HSI}$ & $\begin{array}{l}29.9(26.9- \\
33.7)\end{array}$ & $\begin{array}{l}31.6(29.0- \\
34.1)\end{array}$ & $\begin{array}{l}33.3(29.5- \\
37.5)\end{array}$ & $\begin{array}{l}38.0(32.7- \\
41.2)\end{array}$ & $<0.001$ \\
\hline AST (U/L) & $\begin{array}{l}16.0(14.0- \\
21.3)\end{array}$ & $\begin{array}{l}18.0(16.0- \\
21.0)\end{array}$ & $\begin{array}{l}21.0(19.0- \\
26.0)\end{array}$ & $\begin{array}{l}25.0(21.8- \\
29.3)\end{array}$ & $<0.001$ \\
\hline $\mathrm{ALT}(\mathrm{U} / \mathrm{L})$ & $12.0(9.0-16.0)$ & $\begin{array}{l}15.0(12.0- \\
19.0)\end{array}$ & $\begin{array}{l}19.0(17.0- \\
29.3)\end{array}$ & $\begin{array}{l}28.5(21.0- \\
38.3)\end{array}$ & $<0.001$ \\
\hline Y-GTP (U/L) & $\begin{array}{l}16.0(11.3- \\
21.5)\end{array}$ & $\begin{array}{l}19.0(15.0- \\
24.3)\end{array}$ & $\begin{array}{l}24.0(19.0- \\
34.0)\end{array}$ & $\begin{array}{l}29.0(21.5- \\
44.5)\end{array}$ & $<0.001$ \\
\hline Hypertension & 40 & 41 & 46 & 44 & 0.252 \\
\hline
\end{tabular}




\begin{tabular}{|llllll|}
\hline XOR & Quartile 1 & Quartile 2 & Quartile 3 & Quartile 4 & $\begin{array}{l}\text { P for } \\
\text { trend }\end{array}$ \\
\hline Variables & $\mathbf{( N = 5 6 )}$ & $\mathbf{( N = 5 5 )}$ & $\mathbf{( N = 5 6 )}$ & $\mathbf{( N = 5 6 )}$ & \\
\hline Diabetes & 8 & 11 & 13 & 19 & 0.013 \\
\hline Dyslipidemia & 29 & 37 & 34 & 45 & 0.005 \\
\hline
\end{tabular}

Based on the plasma XOR activity, the subjects were divided into quadrants. Clinical parameters and the proportion of comorbidities between quartiles were examined using Jonckheere-Terpstra test or Cochran-Armitage test.

BMI, body mass index; AC, abdominal circumference; SFA, subcutaneous fat area; visceral fat area, VFA; $\mathrm{HbA1c}$, hemoglobin A1c; HOMA-R, homeostasis model assessment ratio; T-Chol, total-cholesterol; TG, triglycerides; HDL-Chol, high-density lipoprotein-cholesterol; UA, uric acid; L/S, liver-to-spleen; HSI, hepatic steatosis index; AST, aspartate transaminase; ALT, alanine transaminase: $\gamma$-GTP, $\mathrm{Y}$-glutamyl transpeptidas.

Participants were divided into quartiles based on serum UA levels (Table 3). Similar to XOR activity, BMI, AC, SFA, VFA, AST, ALT, and Y-GTP tended to increase with increasing UA levels. HOMA-R, but not HbA1c, tended to increase with increasing UA levels. The proportion of the L/S ratio $<1.1$ and $\mathrm{HSI}>36.0$ for each serum UA level is presented in Fig. 1. The results indicated that the proportion of the L/S ratio $<1.1$ and $\mathrm{HSI}>36.0$ tended to increase with increasing serum UA level. 
Table 3

Differences in clinical parameters among quadrants of serum uric acid (UA) levels

\begin{tabular}{|c|c|c|c|c|c|}
\hline $\begin{array}{l}\text { UA } \\
\text { Variables }\end{array}$ & $\begin{array}{l}\text { Quartile } 1 \\
(\mathrm{~N}=54)\end{array}$ & $\begin{array}{l}\text { Quartile } 2 \\
(N=57)\end{array}$ & $\begin{array}{l}\text { Quartile } 3 \\
(N=56)\end{array}$ & $\begin{array}{l}\text { Quartile } 4 \\
(N=56)\end{array}$ & $\begin{array}{l}\text { P for } \\
\text { trend }\end{array}$ \\
\hline Female : Male & $45: 9$ & $41: 16$ & $32: 24$ & $24: 32$ & $<0.001$ \\
\hline Age (years) & $70(57-75)$ & $66(60-71)$ & $64(51-70)$ & $65(51-71)$ & 0.030 \\
\hline BMI $\left(\mathrm{kg} / \mathrm{m}^{2}\right)$ & $\begin{array}{l}21.9(19.5- \\
24.3)\end{array}$ & $\begin{array}{l}22.8(20.6- \\
25.0)\end{array}$ & $\begin{array}{l}24.3(21.7- \\
28.7)\end{array}$ & $\begin{array}{l}25.4(22.5- \\
28.9)\end{array}$ & $<0.001$ \\
\hline $\mathrm{AC}(\mathrm{cm})$ & $\begin{array}{l}81.2(71.4- \\
86.3)\end{array}$ & $\begin{array}{l}80.8(74.5- \\
86.6)\end{array}$ & $\begin{array}{l}86.3(80.5- \\
92.8)\end{array}$ & $\begin{array}{l}88.0(81.2- \\
98.4)\end{array}$ & $<0.001$ \\
\hline SFA $\left(\mathrm{cm}^{2}\right)$ & $\begin{array}{l}146.2(94.7- \\
207.8)\end{array}$ & $\begin{array}{l}142.8(102.4- \\
186.5)\end{array}$ & $\begin{array}{l}187.6(130.2- \\
226.6)\end{array}$ & $\begin{array}{l}173.6(122.1- \\
282.0)\end{array}$ & $<0.001$ \\
\hline $\operatorname{VFA}\left(\mathrm{cm}^{2}\right)$ & $\begin{array}{l}60.4(33.7- \\
85.3)\end{array}$ & $\begin{array}{l}79.5(45.1- \\
100.7)\end{array}$ & $\begin{array}{l}87.6(59.4- \\
124.6)\end{array}$ & $\begin{array}{l}110.0(85.4- \\
145.4)\end{array}$ & $<0.001$ \\
\hline HbA1c (\%) & $5.7(5.5-6.3)$ & $5.8(5.6-6.1)$ & $5.8(5.6-6.4)$ & $5.8(5.6-6.1)$ & 0.469 \\
\hline HOMA-R & $1.1(0.9-1.6)$ & $1.1(0.8-1.7)$ & $1.7(1.1-2.8)$ & $1.9(1.3-2.6)$ & $<0.001$ \\
\hline $\begin{array}{l}\text { T-Chol } \\
\text { (mg/dL) }\end{array}$ & $\begin{array}{l}188.5(168.5- \\
208.0)\end{array}$ & $\begin{array}{l}194.0(181.0- \\
214.0)\end{array}$ & $\begin{array}{l}197.5(174.8 \\
-214.5)\end{array}$ & $\begin{array}{l}194.5(174.3 \\
-217.3)\end{array}$ & 0.201 \\
\hline TG (mg/dL) & $\begin{array}{l}94.5(73.8- \\
123.8)\end{array}$ & $\begin{array}{l}97.0(66.0- \\
142.0)\end{array}$ & $\begin{array}{l}111.5(83.0- \\
172.8)\end{array}$ & $\begin{array}{l}129.0(101.8- \\
176.8)\end{array}$ & $<0.001$ \\
\hline $\begin{array}{l}\mathrm{HDL}-\mathrm{Chol} \\
(\mathrm{mg} / \mathrm{dL})\end{array}$ & $\begin{array}{l}64.5(55.0- \\
75.8)\end{array}$ & $\begin{array}{l}58.0(51.0- \\
71.0)\end{array}$ & $\begin{array}{l}57.0(48.0- \\
66.5)\end{array}$ & $\begin{array}{l}51.5(42.0- \\
61.3)\end{array}$ & $<0.001$ \\
\hline $\begin{array}{l}\text { XOR activity } \\
\text { (pmol/h/mL) }\end{array}$ & $\begin{array}{l}27.6(19.2- \\
45.9)\end{array}$ & $\begin{array}{l}39.6(25.6- \\
71.6)\end{array}$ & $\begin{array}{l}54.6(30.9- \\
92.6)\end{array}$ & $\begin{array}{l}59.0(26.8- \\
93.8)\end{array}$ & $<0.001$ \\
\hline $\mathrm{UA}(\mathrm{mg} / \mathrm{dL})$ & $3.9(3.3-4.2)$ & $4.9(4.7-5.1)$ & $5.6(5.4-6.0)$ & $6.6(6.5-7.1)$ & $<0.001$ \\
\hline L/S ratio & $1.4(1.3-1.5)$ & $1.4(1.2-1.5)$ & $1.3(1.2-1.4)$ & $1.3(1.1-1.4)$ & $<0.001$ \\
\hline HSI & $\begin{array}{l}31.2(28.3- \\
34.5)\end{array}$ & $\begin{array}{l}31.3(28.4- \\
34.8)\end{array}$ & $\begin{array}{l}35.7(30.0- \\
39.6)\end{array}$ & $\begin{array}{l}34.7(31.6- \\
39.8)\end{array}$ & $<0.001$ \\
\hline AST (U/L) & $\begin{array}{l}19.0(15.0- \\
24.0)\end{array}$ & $\begin{array}{l}20.0(16.0- \\
22.0)\end{array}$ & $\begin{array}{l}21.5(17.8- \\
26.0)\end{array}$ & $\begin{array}{l}22.0(17.0- \\
26.3)\end{array}$ & 0.020 \\
\hline ALT (U/L) & $\begin{array}{l}15.0(11.0- \\
21.0)\end{array}$ & $\begin{array}{l}16.0(12.0- \\
22.0)\end{array}$ & $\begin{array}{l}21.0(15.5- \\
34.0)\end{array}$ & $\begin{array}{l}21.0(14.8- \\
33.0)\end{array}$ & 0.001 \\
\hline Y-GTP (U/L) & $\begin{array}{l}18.0(12.0- \\
25.0)\end{array}$ & $\begin{array}{l}20.0(14.8- \\
27.3)\end{array}$ & $\begin{array}{l}22.0(17.0- \\
30.5)\end{array}$ & $\begin{array}{l}27.5(20.0- \\
40.8)\end{array}$ & $<0.001$ \\
\hline Hypertension & 38 & 37 & 50 & 46 & 0.018 \\
\hline
\end{tabular}




\begin{tabular}{|llllll|}
\hline UA & Quartile 1 & Quartile 2 & Quartile 3 & Quartile 4 & $\begin{array}{l}\mathbf{P} \text { for } \\
\text { trend }\end{array}$ \\
\hline Variables & $(\mathbf{N}=\mathbf{5 4 )}$ & $\mathbf{( N = 5 7 )}$ & $\mathbf{( N = 5 6 )}$ & $\mathbf{( N = 5 6 )}$ & \\
\hline Diabetes & 13 & 11 & 14 & 13 & 0.892 \\
\hline Dyslipidemia & 35 & 33 & 40 & 37 & 0.536 \\
\hline
\end{tabular}

Based on the serum UA levels, the subjects were divided into quadrants. Jonckheere-Terpstra test or Cochran-Armitage test were conducted to assess trends in individual clinical parameters among quadrants.

BMI, body mass index; AC, abdominal circumference; SFA, subcutaneous fat area; visceral fat area, VFA; $\mathrm{HbA1c}$, hemoglobin A1c; HOMA-R, homeostasis model assessment ratio; T-Chol, total-cholesterol; TG, triglycerides; HDL-Chol, high-density lipoprotein-cholesterol; XOR, xanthine oxidoreductase; L/S, liver-tospleen; HSI, hepatic steatosis index; AST, aspartate transaminase; ALT, alanine transaminase.

\section{Association of the UA levels and XOR activity with hepatic steatosis}

An ordinal logistic regression analysis with the L/S ratio as the objective variable and UA levels as the explanatory variable indicated that UA levels had the crude odds ratio (OR) of 1.454 [95\% confidence interval (CI): $1.159-1.824, \mathrm{P}<0.001$ ] (Table 4). The similar analysis showed that the crude OR for XOR activity per $10 \mathrm{pmol} / \mathrm{h} / \mathrm{mL}$ of 1.080 (95\% Cl: $1.039-1.123, \mathrm{P}<0.001)$. Thus, both UA and XOR activity were associated with a lower L/S ratio. Next, since hepatic steatosis, hyperuricemia, and XOR activity were all associated with insulin resistance, we performed a logistic regression analysis with UA, XOR activity, and HOMA-R as explanatory variables in model 1 . The results indicated that XOR activity and HOMA-R were associated with a lower L/S ratio, with OR for XOR activity per $10 \mathrm{pmol} / \mathrm{h} / \mathrm{mL}$ of 1.052 (95\% Cl: 1.013-1.093, $\mathrm{P}=0.009$ ) and OR for HOMA-R of 1.593 (95\% Cl: $1.230-2.062, \mathrm{P}<0.001$ ), whereas UA was no longer associated with a lower L/S ratio, with OR of 1.228 (95\% Cl: $0.960-1.570, P=0.102)$. Furthermore, since NAFLD is known to be associated with obesity, hypertension, dyslipidemia, and diabetes mellitus $[1,3,14]$, we performed a logistic regression analysis adjusted for age, gender, and presence of hypertension, dyslipidemia, and diabetes mellitus in model 3 . The results indicated that XOR activity was associated with a lower L/S ratio with OR for XOR activity per $10 \mathrm{pmol} / \mathrm{h} / \mathrm{mL}$ of 1.047 (95\% Cl: $1.009-1.086, P=0.016)$, whereas UA was not associated with a lower $L / S$ ratio with OR of $1.047(95 \%$ Cl: $0.800-1.370, P=0.737)$. 
Table 4

Logistic regression analyses of the factors associated with liver-to-spleen (L/S) ratio

\begin{tabular}{|c|c|c|c|c|c|c|c|c|}
\hline & L/S ratio & & & & & & & \\
\hline & & & $\begin{array}{l}\text { Model } 1 \\
(n=216)\end{array}$ & & $\begin{array}{l}\text { Model } 2 \\
(\mathrm{n}=216)\end{array}$ & & $\begin{array}{l}\text { Model } 3 \\
(n=216)\end{array}$ & \\
\hline & $\begin{array}{l}\text { Crude OR } \\
(95 \% \mathrm{Cl})\end{array}$ & $P$ & $\begin{array}{l}\text { OR } \\
(95 \% \mathrm{Cl})\end{array}$ & $\mathrm{P}$ & $\begin{array}{l}\text { OR } \\
(95 \% \mathrm{Cl})\end{array}$ & $P$ & $\begin{array}{l}\text { OR } \\
(95 \% \mathrm{Cl})\end{array}$ & $P$ \\
\hline UA & $\begin{array}{l}1.454 \\
(1.159- \\
1.824)\end{array}$ & $\begin{array}{l}< \\
0.001\end{array}$ & $\begin{array}{l}1.228 \\
(0.960- \\
1.570)\end{array}$ & 0.102 & $\begin{array}{l}1.128 \\
(0.875- \\
1.454)\end{array}$ & 0.354 & $\begin{array}{l}1.047 \\
(0.800- \\
1.370)\end{array}$ & 0.737 \\
\hline $\begin{array}{l}\text { XOR activity } \\
\text { per } 10 \\
\text { pmol/h/mL }\end{array}$ & $\begin{array}{l}1.080 \\
(1.039- \\
1.123)\end{array}$ & $\begin{array}{l}< \\
0.001\end{array}$ & $\begin{array}{l}1.052 \\
(1.013- \\
1.093)\end{array}$ & 0.009 & $\begin{array}{l}1.047 \\
(1.009- \\
1.086)\end{array}$ & 0.016 & $\begin{array}{l}1.047 \\
(1.009- \\
1.086)\end{array}$ & 0.016 \\
\hline HOMA-R & $\begin{array}{l}1.836 \\
(1.437- \\
2.347)\end{array}$ & $<.001$ & $\begin{array}{l}1.593 \\
(1.230- \\
2.062)\end{array}$ & $\begin{array}{l}< \\
0.001\end{array}$ & $\begin{array}{l}1.411 \\
(1.070- \\
1.860)\end{array}$ & 0.015 & $\begin{array}{l}1.407 \\
(1.050- \\
1.884)\end{array}$ & 0.022 \\
\hline BMI & $\begin{array}{l}1.190 \\
(1.117- \\
1.268)\end{array}$ & $\begin{array}{l}< \\
0.001\end{array}$ & & & $\begin{array}{l}1.102 \\
(1.022- \\
1.188)\end{array}$ & 0.012 & $\begin{array}{l}1.087 \\
(1.006- \\
1.174)\end{array}$ & 0.034 \\
\hline
\end{tabular}

In model 1, an ordinal logistic regression analysis was performed with L/S ratio as the objective variable and serum uric acid (UA) levels, plasma xanthine oxidoreductase (XOR) activity, and homeostasis model assessment ratio (HOMA-R) as explanatory variables. In Model 2, body mass index (BMI) was added as an explanatory variable. In Model 3 , logistic regression analysis was performed adjusting for age, gender, and presence of hypertension, dyslipidemia, and diabetes mellitus.

The results of $\mathrm{HSI}$ as a dependent factor are presented in Table 5. Similarly to the L/S ratio, XOR activity was associated with increased HSI with OR for XOR activity per $10 \mathrm{pmol} / \mathrm{h} / \mathrm{mL}$ of $1.138(95 \% \mathrm{Cl}$ : $1.064-$ $1.217, \mathrm{P}<0.001)$. On the contrary, UA value was not significantly associated with increased $\mathrm{HSI}$, with OR of $1.360(95 \% \mathrm{Cl}: 1.000-1.850, \mathrm{P}=0.050)$. 
Table 5

Logistic regression analyses of the factors associated with hepatic steatosis index (HSI)

\begin{tabular}{|lllll|}
\hline \multicolumn{1}{|l|}{ HSI } & & & \\
& & & & \\
& & & Model 1 \\
$(\mathrm{n}=216)$ & \\
& & & & \\
& Crude OR & $\mathrm{P}$ & $\mathrm{OR}$ & $\mathrm{P}$ \\
& $(95 \% \mathrm{Cl})$ & & $(95 \% \mathrm{Cl})$ & \\
\hline UA & 1.620 & $<0.001$ & 1.360 & 0.050 \\
& $(1.250-2.100)$ & & $(1.000-1.850)$ & \\
XOR activity & 1.198 & $<0.001$ & 1.138 & $<0.001$ \\
per 10 pmol/h/mL & $(1.116-1.287)$ & & $(1.064-1.217)$ & \\
\hline HOMA-R & 2.226 & $<0.001$ & 1.747 & 0.001 \\
& $(1.623-3.051)$ & & $(1.243-2.454)$ & \\
\hline
\end{tabular}

A logistic regression analysis was performed for $\mathrm{HSI}$, and $\mathrm{HSI}>36.0$ as high values. In Model 1 , serum uric acid (UA) levels, plasma xanthine oxidoreductase (XOR) activity, and homeostasis model assessment ratio (HOMA-R) were used as explanator

\section{Discussion}

In this study, we investigated the relationship between XOR activity measured accurately using LC/TQMS and indices of hepatic steatosis such as the L/S ratio and HSI in humans. We demonstrate that plasma XOR activity was significant risk factors of hepatic steatosis as assessed by the L/S ratio and HSI, independent of insulin resistance index and serum UA levels.

The prevalence of NAFLD has been reported to be increasing worldwide [1-4]. Insulin resistance is important as a major risk factor for NAFLD $[1,14]$. In this study, we demonstrated that hepatic steatosis is related to HOMA-R. Insulin resistance promotes the transfer of free fatty acids from adipose tissue to the liver [29-31]. Furthermore, insulin resistance stimulates sterol receptor-binding protein 1c (SREBP-1c), a transcription factor that controls the synthesis of fatty acids and triglycerides in the liver, leading to hepatic steatosis [32-36]. Thus, insulin resistance is involved in the development of NAFLD through various mechanisms. Furthermore, NAFLD is also known to exacerbate insulin resistance through direct and indirect mechanisms [32-37].

Although it is known that there is a correlation between insulin resistance and XOR activity [21], the results of this study indicate that plasma XOR activity is associated with hepatic steatosis independently of insulin resistance. It has been reported that XOR activity is increased in NAFLD model mice and that 
administration of XOR activity inhibitors improves high-fat-diet-induced hepatic steatosis [24, 25]. Various mechanisms can be considered through which XOR activity promotes the development of hepatic steatosis. The nucleotide-binding oligomerization domain-like receptor family, pyrin domain-containing 3 (NLRP3), forms the NLRP3 inflammasome in response to free fatty acids and stress. NLRP3 is involved in the pathogenesis of NAFLD by inducing the production of Interleukin (IL)-1 $\beta$ and IL-18 [38]. XOR activity and intrahepatic UA have been reported to activate NLRP3 [24]. In addition, C-Jun N-terminal kinase (JNK) is a protein kinase that is activated in response to oxidative stress. XOR activity induces oxidative stress and activates JNK, and the activated JNK has been reported to inhibit insulin signaling and induce hepatic steatosis $[39,40]$.

UA itself has been reported to induce hepatic lipid accumulation by inducing mitochondrial oxidative stress and insulin resistance [41, 42]. In fact, it has been reported that hyperuricemia is associated with the development and progression of $\operatorname{NAFLD}[17,18,43]$. Contrarily, our study demonstrated that plasma XOR activity, rather than serum UA levels, was associated with hepatic steatosis.

There is a study in which the XOR inhibitors, febuxostat, and allopurinol were administered to mice models of NAFLD. In that study, the authors reported that febuxostat significantly reduced hepatic XOR activity and significantly improved insulin resistance and lipid peroxidation in the liver, even though blood UA levels were similarly reduced in both febuxostat and allopurinol [39]. This result, similarly to that in our study, suggests that XOR activity plays a more important role in hepatic steatosis than UA levels. It has been reported that XOR activity is more related to vascular endothelial dysfunction than blood UA level $[44,45]$. Because XOR activity is involved in both hyperuricemia and the development of NAFLD, its management is potentially important in clinical settings $[39,46]$.

In this study, we used the L/S ratio to evaluate hepatic steatosis, which has been reported to indicate moderate or severe fatty liver with a sensitivity of $93 \%$ [9]. In the study of Westerners, L/S ratio $<0.9$ is considered to indicate the presence of moderate to severe fatty liver [9]. In Japan, a study using liver transplant donors reported that the L/S ratio > 1.1 was sufficient to rule out moderate or severe fatty liver [12]. Furthermore, when liver biopsies were performed to histologically evaluate the liver and compared with the L/S ratio, it was reported that the L/S ratio cutoff value for detecting clinically problematic fatty liver was over 1.1, and the L/S ratio assuming absence of hepatic steatosis was over 1.296 [11]. However, it has been reported that it is difficult to adequately assess mild fatty liver using the L/S ratio [9]. Therefore, cases of mild fatty liver may be included in cases with $L / S$ ratio $\geq 1.1$, and further investigation is considered necessary in the future.

In a case-control study conducted in Korea, it was reported that HSI was useful in predicting NAFLD and that approximately $90 \%$ of cases could be properly diagnosed [13]. In this study, we used NAFLD fibrosis score (NFS) and the FIB-4 index to evaluate the progression of hepatic fibrosis [47, 48], but only 5 cases with L/S ratio $<1.1$ had high NFS, and only 4 patients had a high FIB-4 index, and the significant relationship was not observed between hepatic fibrosis and XOR activity. In this study, liver biopsy was not performed because of its high invasiveness. However, a prospective study including liver biopsy is 
necessary to examine the relationship more accurately between NAFLD onset and progression and XOR activity.

The limitation of this study is that it was a single-center, cross-sectional analysis. In this study, we used the L/S ratio and HSI as indices of hepatic steatosis and NFS and the FIB-4 index as indices of hepatic fibrosis progression, but liver biopsy is necessary for diagnosis of fatty liver, and further studies are required. Furthermore, a prospective study is required to further clarify the relationship between XOR and hepatic steatosis.

In conclusion, the results of this study indicated that plasma XOR activity is associated with hepatic steatosis independently of insulin resistance and serum UA levels. Thus, XOR activity may be potentially involved in hepatic steatosis in humans.

\section{Methods}

\section{Study design and participants.}

This cross-sectional analysis was conducted as part of the Hyogo Sleep Cardio-Autonomic Atherosclerosis (HSCAA) study [49-51]. In summary, the HSCAA study is a single-center cohort study which aims to investigate the interrelationships among sleep disorders, autonomic neuropathy, metabolic diseases, and atherosclerotic diseases [49-51]. The HSCAA study included patients aged 20 years and older with one or more cardiovascular risk factors (obesity, smoking, presence of cardiovascular event history, hypertension, dyslipidemia, diabetes mellitus, chronic kidney disease) and being treated at the Hyogo College of Medicine Hospital.

Since we started XOR measurements from 2018 for the subjects who were registered or followed in the HSCAA study, this cross-sectional study included 310 patients, from January 2018 to July 2021, who consented abdominal CT examinations. In the end, 223 patients were analyzed in the present study after excluding 87 with alcoholic habits ( $>30 \mathrm{~g} /$ day for males and $>20 \mathrm{~g} /$ day for females), autoimmune hepatitis, viral hepatitis, and under treatment with XOR inhibitors.

The HSCAA study has been approved by the Ethics Committee of Hyogo College of Medicine Hospital (Approval No. 2351). Written informed consent was obtained from all subjects and the study was conducted in full accordance with the Declaration of Helsinki. The present study protocol was approved by the Ethics Committee of Hyogo College of Medicine Hospital (Approval No. 3601) and performed with an opt-out option, as explained in instructions posted on the website of the hospital. All methods in our study were performed in accordance with the relevant guidelines and regulations.

\section{Visceral fat area and subcutaneous fat area}

CT was performed using SIEMENS SOMATOM Definition AS+ or SOMATOM Definition H (Siemens Healthcare $\mathrm{GmbH}$, Erlangen, Germany) with $10 \mathrm{~mm}$ slices. We evaluated the visceral fat area (VFA), 
subcutaneous fat area (SFA), and waist circumference using Ziostation 2 (AMIN Ltd., Tokyo, Japan). The abdominal circumference (AC) was measured at the umbilical height.

\section{Hepatic steatosis and liver fibrosis}

The L/S ratio and HSI were used to evaluate hepatic steatosis. Hepatic and splenic attenuation values were measured on non-contrast-CT scans using four circular region-of-interest (ROI) cursors in the liver and two in the spleen. In the liver, four ROls were located in each of the right lobe and the left lobe of the liver. All measurements were manually obtained in regions of uniform parenchymal attenuation, with care being taken to avoid vessels, artifacts, and other areas that might have spuriously increased or decreased measurements. Calculation of the L/S ratio was as follow: $\mathrm{L} / \mathrm{S}$ ratio $=($ Average attenuation value of the liver) / (Average attenuation value of the spleen) [9-12]. HSI was calculated from ALT, AST, BMI, gender, and the presence of diabetes mellitus [13].

In addition, the NAFLD fibrosis score (NFS) and the Fibrosis-4 (Fib-4) index were calculated to predict the progression of liver fibrosis in patients with L/S ratio $<1.1[47,48]$. NFS was calculated from age, BMI, AST, ALT, the presence of glucose intolerance, platelet count, and albumin [47]. It has been reported that by applying the high cutoff score (NFS $>0.676$ ), the presence of advanced fibrosis could be diagnosed with high accuracy [47]. The FIB-4 index was calculated from age, AST, ALT, and platelet count. It has been reported that its cutoff value $<1.45$ can exclude hepatic fibrosis, and its cutoff value $>3.25$ can predict hepatic fibrosis [52].

\section{Plasma XOR activity measurement}

The assay protocol of XOR activity in humans was reported previously [26-28]. In brief, $100 \mu \mathrm{L}$ of plasma samples (purified by Sephadex G25 resin) were mixed with a Tris buffer $(\mathrm{pH} 8.5)$ containing $\left[{ }^{13} \mathrm{C}_{2},{ }^{15} \mathrm{~N}_{2}\right]$ xanthine as a substrate, $\mathrm{NAD+}$, and $\left[{ }^{13} \mathrm{C}_{3},{ }^{15} \mathrm{~N}_{3}\right]$ UA as an internal standard. The mixtures were incubated at $37^{\circ} \mathrm{C}$ for $90 \mathrm{~min}$. Subsequently, the mixtures were mixed with $500 \mu \mathrm{L}$ of methanol and centrifuged at $2,000 \times \mathrm{g}$ for $15 \mathrm{~min}$ at $4^{\circ} \mathrm{C}$. The supernatants were transferred to new tubes and dried using a centrifugal evaporator. The residues were reconstituted with $150 \mu \mathrm{L}$ of distilled water, filtered through an ultrafiltration membrane, and measured using LC/TQMS. LC/TQMS comprised a Nano Space SI-2 LC system (Shiseido Co., Ltd., Tokyo, Japan) and a TSQ Triple Quadrupole LC-MS system (ThermoFisher Scientific $\mathrm{GmbH}$, Bremen, Germany) equipped with an ESI interface. Calibration standard samples of $\left[{ }^{13} \mathrm{C}_{2},{ }^{15} \mathrm{~N}_{2}\right]$ UA were also measured, and the amounts of production were quantitated from the calibration curve. XOR activities were expressed in $\mathrm{pmol} / \mathrm{mL} / \mathrm{h}$ [26-28].

\section{Other parameters}

At the same time as that for the CT scan, blood samples were taken for AST, ALT, UA, fasting blood glucose, immunoreactive insulin, total cholesterol (T-Chol), high-density lipoprotein cholesterol (HDLChol), and TG. Serum UA levels were measured using the uricase/peroxidase technique with an 
autoanalyzer using UA (Pureauto S UA Sekisui Medical, Ltd., Tokyo, Japan). Height, weight, and blood pressure were also measured.

Type 2 diabetes was diagnosed based on results showing fasting plasma glucose $\geq 126 \mathrm{mg} / \mathrm{dL}$, causal plasma glucose $\geq 200 \mathrm{mg} / \mathrm{dL}$, or 2-h plasma glucose $\geq 200 \mathrm{mg} / \mathrm{dl}$ during a $75-\mathrm{g}$ oral glucose tolerance test, or previous therapy for diabetes [53]. Hypertension was defined as systolic blood pressure $\geq 140$ $\mathrm{mmHg}$, diastolic blood pressure $\geq 90 \mathrm{mmHg}$, or taking treatment for hypertension. We defined dyslipidemia as the presence of LDL-C $\geq 140 \mathrm{mg} / \mathrm{dL}, \mathrm{HDL}-\mathrm{C} \leq 40 \mathrm{mg} / \mathrm{dL}$, TG level $\geq 150 \mathrm{mg} / \mathrm{dL}$, or taking treatment for dyslipidemia.

\section{Statistical analysis}

The results were presented as median (interquartile range), unless otherwise stated. We used the Jonckheere-Terpstra test to compare the trend of data between three or more groups. The CochranArmitage test was used for the trend of the ratio between three or more groups.

Hepatic steatosis was graded as follows: with hepatic steatosis (L/S ratio $<1.1)$ [11, 12], without hepatic steatosis (L/S ratio >1.296), and intermediate $(\mathrm{L} / \mathrm{S}$ ratio $=1.1-1.296)$ [11]. In model 1 , an ordinal logistic regression analysis was performed with $\mathrm{L} / \mathrm{S}$ ratio as the objective variable and serum UA levels, plasma XOR activity, and HOMA-R as explanatory variables. In Model 2, BMI was added as an explanatory variable. In Model 3, we used an ordinal logistic regression analysis, and the L/S ratio was used as the objective variable; UA, XOR activity, and the HOMA-R were used as the explanatory variables, adjusted for age, gender, and components for Japanese diagnostic criteria of metabolic syndrome (AC, blood pressure, plasma glucose, HDL, and TG). Furthermore, $\mathrm{HSI}>36.0$ were defined as high values [13], and logistic regression analyses was performed with $\mathrm{HSI}$ as the objective variables and UA, XOR, and HOMA-R as explanatory variables.

Statistical analyses were conducted using the BellCurve software version 2.15 (Social Survey Research Information Co., Ltd., Tokyo, Japan), with $\mathrm{P}<0.05$ indicating statistical significance.

\section{Declarations}

\section{Acknowledgments}

The authors thank the subjects who participated in this study and all the physicians and the co-medical staff at Hyogo college medicine hospital for their support in collecting the data.

\section{Author Contributions}

C.Y, Y.K, T.T, and H.K were engaged in the preparation of the study protocol, the analysis of data, laboratory tests, and the preparation of this article. T.M and T.N were engaged in laboratory test. K.O, M.O, A.Mo, A.Mi, M.K-H, K.K-H, M.K, K.K, and T.S were engaged in the data collection. All authors listed have contributed to the work and approved the final version. 


\section{Competing interests}

While Takayo Murase and Takashi Nakamura are affiliated with Sanwa Kagaku Kenkyusho Co. Ltd., their involvment does not alter our adherence regarding sharing of data and materials. The other authors have no conflicts of interest to declare.

\section{Funding}

The present study was supported in part by Japan Society for The Promotion of Science KAKENHI Grant Number: 21K10511.

\section{References}

1. The Japanese Society of Gastroenterogy and the Japan Society of Hepatology. Evidence-based clinical practice guidelines for nonalcoholic fatty liver disease/nonalcoholic steatohepatitis (2nd ed) (ed. the Japanese Society of Gastroenterogy and the Japan Society of Hepatology.) [in Japanese] 285 (Nankodo, 2020).

2. Chalasani, N. et al. The diagnosis and management of nonalcoholic fatty liver disease: practice guidance from the American Association for the Study of Liver Diseases., 67, 328-357 (2018).

3. Younossi, Z. M. et al. Global epidemiology of nonalcoholic fatty liver disease-meta-analytic assessment of prevalence, incidence, and outcomes., 64, 73-84 (2016).

4. Eguchi, Y. et al. Prevalence and associated metabolic factors of nonalcoholic fatty liver disease in the general population from 2009 to 2010 in Japan: a multicenter large retrospective study. $J$ Gastroenterol, 47, 586-595 (2012).

5. Adams, L. A. et al. The natural history of nonalcoholic fatty liver disease: a population-based cohort study., 129, 113-121 (2005).

6. Ekstedt, M. et al. Long-term follow-up of patients with NAFLD and elevated liver enzymes. Hepatology. 44, 865 - 73(2006).

7. Musso, G., Gambino, R., Cassader, M. \& Pagano, G. Meta-analysis: natural history of non-alcoholic fatty liver disease (NAFLD) and diagnostic accuracy of non-invasive tests for liver disease severity. Ann Med, 43, 617-649 (2011).

8. Ricci, C. et al. Noninvasive in vivo quantitative assessment of fat content in human liver. J Hepatol, 27, 108-113 (1997).

9. Saadeh, S. et al. The utility of radiological imaging in nonalcoholic fatty liver disease., $123,745-750$ (2002).

10. Park, S. H. et al. Macrovesicular hepatic steatosis in living liver donors: use of CT for quantitative and qualitative assessment., 239, 105-112 (2006).

11. Kan, H. et al. Non-invasive assessment of liver steatosis in non-alcoholic fatty liver disease. Hepatol Res, 44, E420-E27 (2014). 
12. Iwasaki, M. et al. Noninvasive evaluation of graft steatosis in living donor liver transplantation. Transplantation, 78, 1501-1505 (2004).

13. Lee, J. H. et al. Hepatic steatosis index: a simple screening tool reflecting nonalcoholic fatty liver disease. Dig Liver Dis, 42, 503-508 (2010).

14. Diehl, A. M. \& Day, C. Cause, pathogenesis, and treatment of nonalcoholic steatohepatitis. N Eng/ J Med, 377, 2063-2072 (2017).

15. NICE guideline. Nonalcoholic fatty liver disease (NAFLD): assessment and management. https://www.nice.org.uk/guidance/ng49/chapter/Recommendations. [Accessed July 1, 2021].

16. Younossi, Z. M. et al. The global epidemiology of NAFLD and NASH in patients with type 2 diabetes: a systematic review and meta-analysis. J Hepatol, 71, 793-801 (2019).

17. Ma, Z. et al. Hyperuricemia precedes non-alcoholic fatty liver disease with abdominal obesity moderating this unidirectional relationship: three longitudinal analyses. Atherosclerosis, 311, 44-51 (2020).

18. Yuan, H. et al. Serum uric acid levels and risk of metabolic syndrome: a dose-response meta-analysis of prospective studies. J Clin Endocrinol Metab, 100, 4198-4207 (2015).

19. Meneshian, A. \& Bulkley, G. B. The physiology of endothelial xanthine oxidase: from urate catabolism to reperfusion injury to inflammatory signal transduction., 9, 161-175 (2002).

20. Berry, C. E. \& Hare, J. M. Xanthine oxidoreductase and cardiovascular disease: molecular mechanisms and pathophysiological implications. J Physiol, 555 (Pt 3), 589-606 (2004).

21. Washio, K. W. et al. Xanthine oxidoreductase activity is correlated with insulin resistance and subclinical inflammation in young humans. Metabolism, 70, 51-56 (2017).

22. Battelli, M. G., Bortolotti, M., Polito, L. \& Bolognesi, A. The role of xanthine oxidoreductase and uric acid in metabolic syndrome. BBA-Molecular Basis of Disease, 1864, 2557-2565 (2018).

23. Kuppusamy, U. R., Indran, M. \& Rokiah, P. Glycaemic control in relation to xanthine oxidase and antioxidant indices in Malaysian Type 2 diabetes patients. Diabet Med, 22, 1343-1346 (2005).

24. Xu, C. et al. Xanthine oxidase in non-alcoholic fatty liver disease and hyperuricemia: one stone hits two birds. J Hepatol, 62, 1412-1419 (2015).

25. Nakatsu, Y. et al. The xanthine oxidase inhibitor febuxostat suppresses development of nonalcoholic steatohepatitis in a rodent model. Am J Physiol Gastrointest Liver Physiol, 309, G42-51 (2015).

26. Murase, T. et al. Xanthine oxidoreductase activity assay in tissues using stable isotope-labeled substrate and liquid chromatography high-resolution mass spectrometry. J Chromatogr $B, 1008$, 189-197 (2016).

27. Murase, T., Oka, M., Nampei, M., Miyachi, A. \& Nakamura, T. A highly sensitive assay for xanthine oxidoreductase activity using a combination of [13C2,15N2] xanthine and liquid chromatography/triple quadrupole mass spectrometry. J Labelled Comp Radiopharm, 59, 214-220 (2016). 
28. Murase, T., Nampei, M., Oka, M., Miyachi, A. \& Nakamura, T. A highly sensitive assay of human plasma xanthine oxidoreductase activity using stable isotope-labeled xanthine and LC/TQMS. $J$ of Chromatogr B Analyt Technol Biomed Life Sci, 1039, 51-58 (2016).

29. Reccia, I. et al. Non-alcoholic fatty liver disease: a sign of systemic disease. Metabolism. 72,94108(2017).

30. Bae, J. C. et al. Impact of nonalcoholic fatty liver disease on insulin resistance in relation to HbA1c levels in nondiabetic subjects. Am J Gastroenterol, 105, 2389-2395 (2010).

31. Watt, M. J., Miotto, P. M., De Nardo, W. \& Montgomery, M. K. The liver as an endocrine organ-linking NAFLD and insulin resistance. Endocr Rev, 40, 1367-1393 (2019).

32. Reccia, I. et al. Non-alcoholic fatty liver disease: a sign of systemic disease. Metabolism, 72, 94-108 (2017).

33. Bae, J. C. et al. Impact of nonalcoholic fatty liver disease on insulin resistance in relation to HbA1c levels in nondiabetic subjects. Am J Gastroenterol, 105, 2389-2395 (2010).

34. Watt, M. J., Miotto, P. M., De Nardo, W. \& Montgomery, M. K. The liver as an endocrine organ-linking NAFLD and insulin resistance. Endocr Rev, 40, 1367-1393 (2019).

35. Khan, R. S., Bril, F., Cusi, K. \& Newsome, P. N. Modulation of insulin resistance in nonalcoholic fatty liver disease., 70, 711-724 (2019).

36. Shimomura, I., Bashmakov, Y. \& Horton, J. D. Increased levels of nuclear SREBP-1c associated with fatty livers in two mouse models of diabetes mellitus. J Biol Chem, 274, 30028-30032 (1999).

37. Samuel, V. T. \& Shulman, G. I. Non-alcoholic fatty liver disease as a nexus of metabolic and hepatic diseases. Cell Metab, 27, 22-41 (2018).

38. Wang, Y. G. et al. The involvement of NLRX1 and NLRP3 in the development of nonalcoholic steatohepatitis in mice. J Chin Med Assoc, 76, 686-692 (2013).

39. Nishikawa, T. et al. Xanthine oxidase inhibition attenuates insulin resistance and diet-induced steatohepatitis in mice. Sci Rep, 10, 815 (2020).

40. Czaja, M. J. JNK regulation of hepatic manifestations of the metabolic syndrome. Trends Endocrinol Metab, 21, 707-713 (2010).

41. Sautin, Y. Y., Nakagawa, T., Zharikov, S. \& Johnson, R. J. Adverse effects of the classic antioxidant uric acid in adipocytes: NADPH oxidase-mediated oxidative/nitrosative stress. Am J Physiol Cell Physiol, 293, C584-96 (2007).

42. Lanaspa, M. A. et al. Uric acid induces hepatic steatosis by generation of mitochondrial oxidative stress: potential role in fructose-dependent and -independent fatty liver. J Biol Chem, 287, 4073240744 (2012).

43. Gong, S., Song, J., Wang, L., Zhang, S. \& Wang, Y. Hyperuricemia and risk of nonalcoholic fatty liver disease: a systematic review and meta-analysis. Eur J Gastroenterol Hepatol, 28, 132-138 (2016).

44. George, J., Carr, E., Davies, J., Belch, J. J. F. \& Struthers, A. High-dose allopurinol improves endothelial function by profoundly reducing vascular oxidative stress and not by lowering uric acid., 114, 2508- 
2516 (2006).

45. Washio, K. et al. Xanthine oxidoreductase activity correlates with vascular endothelial dysfunction in patients with type 1 diabetes. Acta Diabetol, 57, 31-39 (2020).

46. Furuhashi, M. New insights into purine metabolism in metabolic diseases: role of xanthine oxidoreductase activity. Am J Physiol Endocrinol Metab, 319, E827-E834 (2020).

47. Angulo, A. et al. The NAFLD fibrosis score: a noninvasive system that identifies liver fibrosis in patients with NAFLD. Hepatology. 45, 847 - 54(2007).

48. McPherson, S., Stewart, S. F., Henderson, E., Burt, A. D. \& Day, C. P. Simple non-invasive fibrosis scoring system can reliably exclude advanced fibrosis in patients with non-alcoholic fatty liver disease., 59, 1265-1269 (2010).

49. Kadoya, M. et al. Plasma brain-derived neurotrophic factor and reverse dipping pattern of nocturnal blood pressure in patients with cardiovascular risk factors. PLoS One, 9, e105977 (2014).

50. Kadoya, M. et al. Associations of Sleep Quality and Awake Physical Activity with Fluctuations in Nocturnal Blood Pressure in Patients with Cardiovascular Risk Factors. PLoS One, 11, e0155116 (2016).

51. Kadoya, M. et al. Serum Macro TSH Level is Associated with Sleep Quality in Patients with Cardiovascular Risks - HSCAA Study. Sci Rep, 7, 44387 (2017).

52. Sterling, R. K. et al. Development of a simple noninvasive index to predict significant fibrosis in patients with HIV/HCV coinfection. Hepatology, 43, 1317-1325 (2006).

53. Haneda, M. et al. Japanese Clinical Practice Guideline for Diabetes 2016. J Diabetes Investig, 9, 657697 (2018).

\section{Figures}


Figure. 1

A.

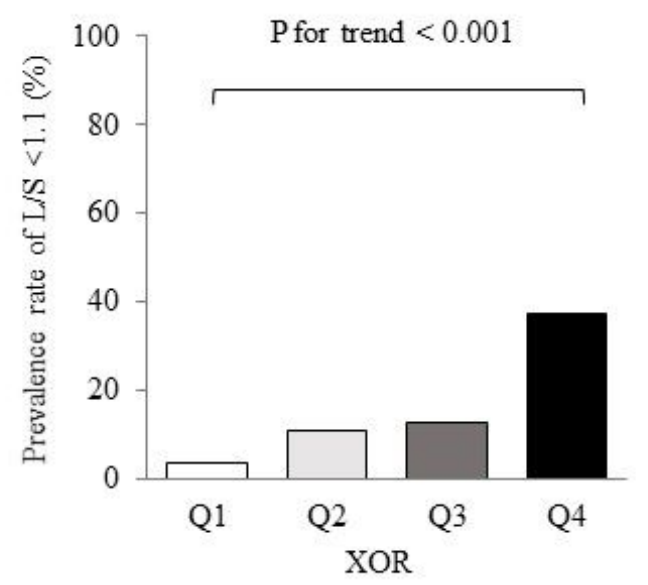

C.

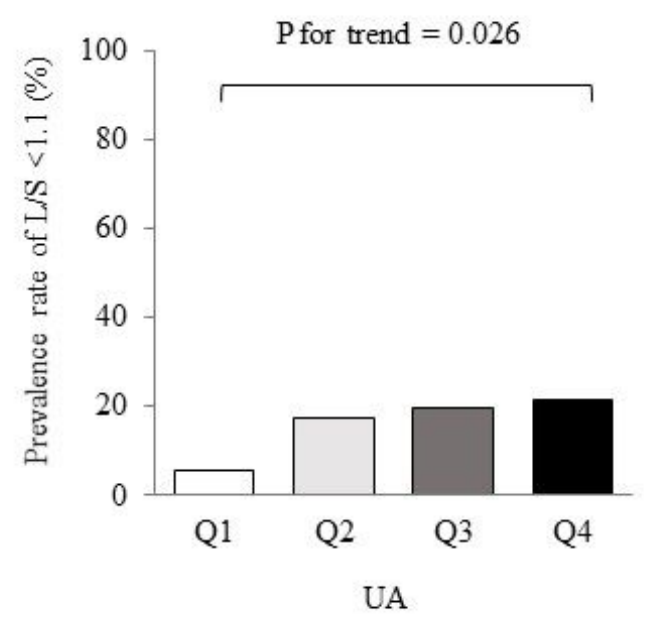

B.

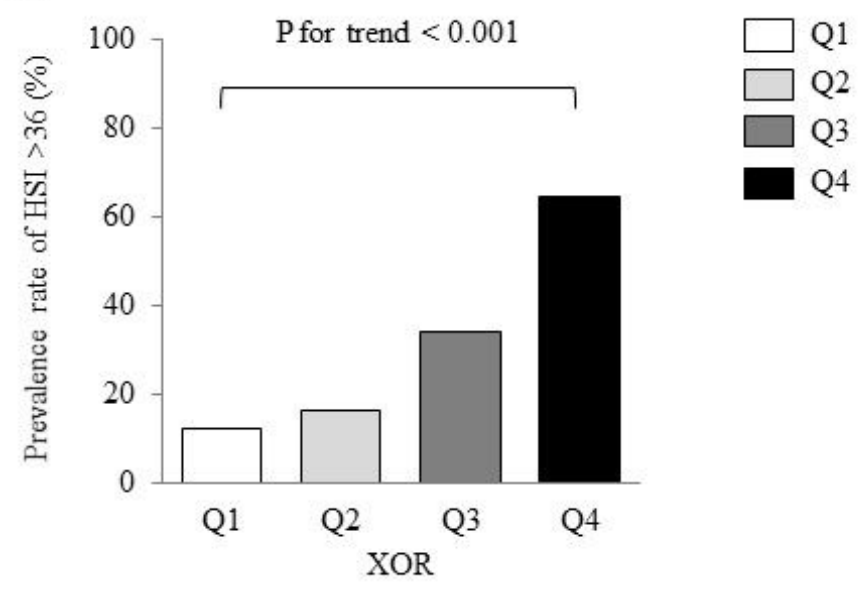

D.

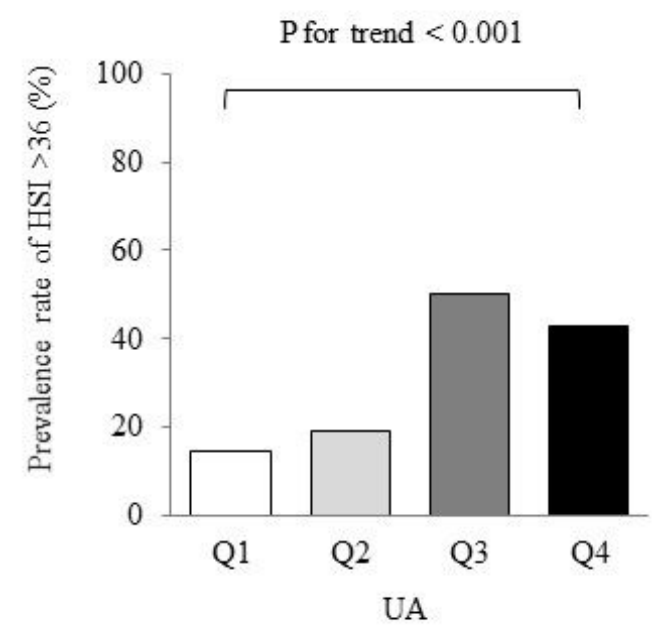

\section{Figure 1}

Comparisons of indices of hepatic steatosis among quadrants of XOR activity or UA levels A. Xanthine oxidoreductase (XOR) activity and prevalence rate of liver-to-spleen (L/S) ratio $<1.1$, B. Uric acid (UA) levels and prevalence rate of $L / S$ ratio $<1.1, C$. XOR and prevalence rate of hepatic steatosis index (HSI) $>36.0$, D. UA and prevalence rate of $\mathrm{HSI}>36.0$. The proportion of hepatic steatosis between quartiles was examined using the Cochran-Armitage test. Abbreviations: Q, quadrant 